\title{
Theoretical investigations of a modified compressed ultrafast photography method suitable for single-shot fluorescence lifetime imaging
}

\author{
YAHUI LI $1,2,4,{ }^{*}$, JINSHOU TIAN ${ }^{1,2,4}$, AND DAVID DAY-UEI LI ${ }^{3}$ \\ ${ }^{1}$ Key Laboratory of Ultra-fast Photoelectric Diagnostics Technology, Xi'an Institute of Optics and Precision Mechanics, Xi'an Shaanxi 710049, People's Republic \\ of China \\ ${ }^{2}$ University of Chinese Academy of Sciences, Beijing 100049, People's Republic of China \\ ${ }^{3}$ Strathclyde Institute of Pharmacy and Biomedical Sciences, University of Strathclyde, GlasgowG4 ORE, United Kingdom \\ ${ }^{4}$ Collaborative Innovation Center of Extreme Optics, Shanxi University, Taiyuan Shanxi 030006, People's Republic of China \\ *Corresponding author: yahuili777@hotmail.com
}

Compiled January 15, 2021

\begin{abstract}
A single-shot fluorescence lifetime imaging (FLIM) method based on the compressed ultrafast photography (CUP) is proposed, named space-restricted CUP (srCUP). srCUP is suitable for imaging objects moving slowly ( $<\sim 150 / \mathrm{M} \mathrm{mm} / \mathrm{s}, M$ is the magnification of the objective lens) in the field of view with the intensity changing within nanoseconds in a measurement window around $10 \mathrm{~ns}$. We used synthetic datasets to explore the performances of srCUP compared with CUP and TCUP (a variant of CUP). srCUP not only provides superior reconstruction performances, but its reconstruction time is also two- and threefold faster than CUP and TCUP, respectively. The lifetime determination performances were assessed by estimating lifetime components, amplitude- and intensity-weighted average lifetimes $\left(\tau_{A}\right.$ and $\left.\tau_{I}\right)$ with the reconstructed scenes using the least square method based on a bi-exponential model. srCUP has the best accuracy and precision for lifetime determinations with a relative bias less than $7 \%$ and a coefficient of variation less than $7 \%$ for $\tau_{A}$ and a relative bias less than $10 \%$ and a coefficient of variation less than $11 \%$ for $\tau_{I}$. (๑) 2021 Optical Society of America
\end{abstract}

http://dx.doi.org/10.1364/ao.XX.XXXXXX

\section{INTRODUCTION}

Fluorescence lifetime imaging (FLIM) [1, 2] is a powerful imaging technique used in life sciences [3-5] and flow diagnosis areas [6-9] to observe the microenvironments of fluorescent molecules, such as $\mathrm{pH}[10,11]$, temperature $[12,13]$, viscosity $[14,15]$, or ion concentrations [16, 17]. FLIM can also access protein conformational changes and protein-protein interactions through Förster Resonance Energy Transfer (FRET) processes $[18,19]$. Compared to fluorescence intensity imaging, FLIM is independent of the laser intensity and fluorophore concentrations. FLIM can be implemented in the time and frequency domains [20]. Time-domain systems are usually based on time-correlated single-photon counting (TCSPC) techniques [21-23], time-gated cameras $[6,7,24]$, or streak cameras (SC) $[25,26]$. The SC method needs a scanning process to obtain two-dimensional (2D) FLIM images; the acquisition is usually slow.

An ultrafast imaging method called the compressed ultrafast photography (CUP) [27] was introduced to enable SCs capturing 2D dynamic scenes in a single-shot. CUP works at a time resolution of 10 ps by compressing a coded 3D scene into a
2D snapshot and then reconstructing the 3D scene by solving an inverse optimization problem. An improved CUP variant was proposed, called the trillion-frame-per-second CUP (TCUP) [28], working at a time resolution of $100 \mathrm{fs}$ by including an unsheared view recorded by an additional camera. Recently, CUP has been further improved for various applications, including high-dimensional optical imaging [29], ultraviolet imaging [30], ultrafast electron diffraction imaging [31], phase-sensitive imaging [32] and fluorescence lifetime imaging [33-35]. For more information about CUP, please refer to the review paper [36].

Two features, the frame rate and the exposure time of a frame, are usually used to assess the speed of an imaging method. A high frame-rate camera can capture multiple frames with short frame intervals, whereas a short exposure time of a frame means a transient scene can be 'frozen' in a short measurement window. A single-shot FLIM method can provide a FLIM image with a short exposure time $(\sim 10 \mathrm{~ns})$ by illuminating an object with a single pulse (single-shot).

For wide-field TCSPC systems [21,37-39], the exposure time of one frame is much longer in comparison, for example around 
$0.01 \mathrm{~s}$ with a $64 \times 64$ SPAD array at a frame rate of $100 \mathrm{fps}$ [40] which means it needs $\sim 0.01 \mathrm{~s}$ to obtain a dataset for evaluating a FLIM image. The dead-time of a TCSPC module limits the allowable photon count rate (< one photon event for every 100 laser pulses) to avoid pile-up effects [41]. If objects move fast, a long exposure time of a frame can lead to a motion blur, or if the microenvironments of objects change rapidly, a long exposure time of a frame will result in loss of time-resolved information. Therefore, developing single-shot FLIM methods is meaningful for studying dynamic events requiring a short exposure time of a frame $(\sim 10 \mathrm{~ns})$. Time-gated cameras can achieve single-shot FLIM by recording time-gated intensity images simultaneously with two ICCDs [7] or a segmented gated optical intensifier [42]. However, single-shot FLIM methods with time-gated cameras (mainly used for fast FLIM contrast imaging) suffer from low lifetime estimation precision for a limited number of time-gated images obtained [43,44]. It is challenging to use time-gated systems to reconstruct full decay profiles. There is no way users can know whether lifetime estimations are correct if there are artefacts in the measurements. For time-resolved signals following a complex decay profile, obtaining full decay profiles are favored for precise lifetime analysis. CUP is much more suitable for this task than time-gated systems [33-35]. However, original CUP techniques were not optimized for FLIM applications. Modifying CUP suitable for FLIM in terms of reconstruction and lifetime determination performances is desirable.

Unlike a typical dynamic scene where objects move unpredictably across the field of view (FoV), biological objects usually move slowly, and only the intensities of signals emitted from objects change rapidly within the measurement window. Fig. 1 shows the flow diagrams of CUP and TCUP. The random coding pattern generated with a digital mirror device (DMD) covers the whole FoV and does not depend on objects' distribution. CUP obtains an SC image with a single-shot excitation, whereas TCUP captures an integrated intensity image and an SC image for reconstruction. In low light conditions, most pixels do not detect enough fluorescence and are prone to noise. The random patterns covering the whole FoV used in traditional CUP and TCUP can contribute to inaccurate lifetime estimations.

The spatial invariance of objects for FLIM within a few milliseconds can be applied to the TCUP system to accelerate the reconstruction and improve the performances. Recently, Ma et al. achieved FLIM of live cells with a CUP scheme and employed an algorithm with a spatial constraint for reconstruction [34]. However, background noise is still included in SC images in their method, which will affect reconstruction and lifetime determination performances.

To eliminate background noise during data acquisition, we proposed a modified TCUP method suitable for FLIM, called space-restricted CUP (srCUP). Instead of coding the whole FoV in a TCUP system, srCUP only codes the area where the integrated intensity larger than an intensity threshold. The pixel noise outside the coded area is effectively excluded in SC images, and the number of the pixels for optimization can be significantly reduced. Synthetic datasets can simulate $3 \mathrm{D}(x, y, t)$ scenes. The reconstruction and lifetime determination performances were explored with CUP, TCUP, and srCUP for comparison.

\section{SYSTEM AND METHODOLOGY}

The proposed srCUP system consists of a fluorescence signal generation system and a modified TCUP system, as shown in Fig. 2. The fluorescence signal generation system contains a

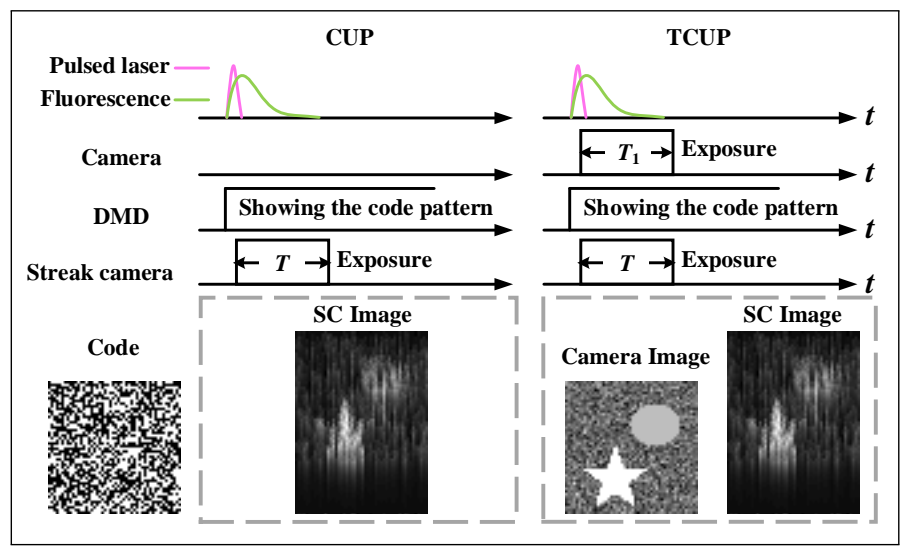

Fig. 1. Flow diagrams of CUP and TCUP.

pulsed laser to generate ultrafast laser pulses, a beam modification module for converting the laser beam into a laser sheet, and an object illuminated by the laser sheet to emit fluorescence. The wavelength of the laser pulse is aligned to the peak absorption spectra of fluorescent molecules in the object. The beam modification module includes a convex lens $\mathrm{L}_{1}$ and a concave lens $\mathrm{L}_{2}$ (to expand the laser beam), a convex cylindrical lens $\mathrm{CL}_{1}$, and a concave cylindrical lens $\mathrm{CL}_{2}$ to compress the expanded beam to form a laser sheet.

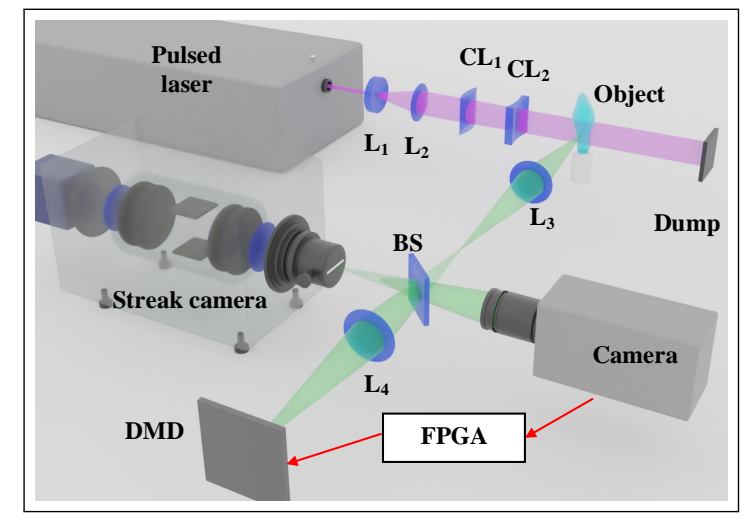

Fig. 2. Illustration of the srCUP system.

The modified TCUP system detects fluorescence signals. The intersection of the laser sheet and the object is in the object plane of the TCUP system. Through the objective lens $\mathrm{L}_{3}$, the signal is directed by a beam splitter (BS) to a camera and a DMD. The DMD codes the signal and then reflects the signal into a SC through the lens $\mathrm{L}_{4}$ and BS. If the magnifications of $\mathrm{L}_{4}$ and the camera lens are 1 , the pixel sizes of the camera, the DMD, and the SC should be the same.

Fig. 3 shows the working principle of the srCUP system. The pulsed laser generates two pluses illuminating objects in the FoV successively with an interval $t_{p}$. The first signal generated with the first pulse is detected only by the camera to obtain an integrated intensity image 1 . The grayscale represents the intensity. Image 1 is sent to a field-programmable gate array (FPGA) to be processed to generate a binary code. The FPGA algorithm for code generation can be implemented in two steps: 1) hard thresholding, with which an image mask is obtained (the pixels with intensities larger than a threshold are set to 1 and the others 0$) ; 2$ ) randomly coding the pixels in the masked 
area with 1 or 0 . The code is then sent from the FPGA to the DMD controller to set the DMD with the same pattern before the second signal arrives at the DMD. The total time for the code setting upon Image 1 is denoted as $t_{c}$. Both the camera and the $\mathrm{SC}$ detect the second signal. The second signal directed to the SC is coded by the DMD showing the code generated with the first pulse. The images obtained with the second pulse, Image 2 and SC Image, are used for reconstruction.

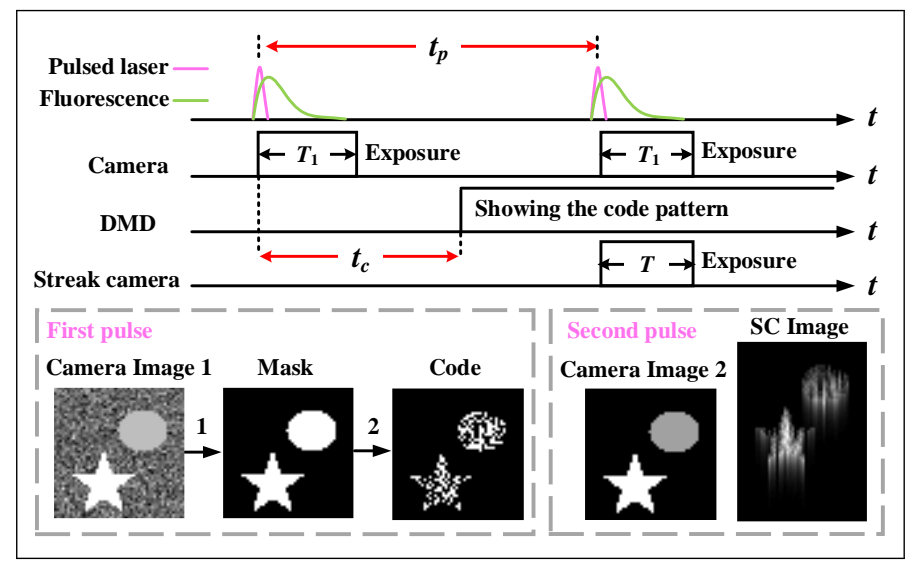

Fig. 3. Working principle of srCUP.

Although two pulses are needed in srCUP, the first one is used to set the DMD code pattern. The camera and SC images used for reconstruction are from a single-shot (the second pulse). Therefore, it is reasonable to call srCUP a single-shot FLIM method.

For general scenes, the camera and the SC's exposures should be the same to adopt the reconstruction algorithm, i.e. $T_{1}=T$. However, for FLIM, a fluorescence signal has a lifetime $\tau$ usually in a few nanoseconds, which means the intensity approaches zero after $t \sim 4 \tau$ with respect to the laser pulse. Therefore, $T_{1}$ and $T$ are not necessarily equal, but they should be larger than $\sim 4 \tau$ to cover most of the decay. The resulting FLIM's time resolution is determined by the maximum lifetime of the object in single-shot schemes. For example, if an object has a lifetime of $2.5 \mathrm{~ns}, T_{1}$ and $T>10 \mathrm{~ns}$, the time resolution is about $10 \mathrm{~ns}$.

The application of srCUP is limited by $t_{c}$. The code setting process includes reading out the intensity image from the camera, generating a code pattern with the FPGA, and controlling the DMD to create the code pattern. $t_{c}$ can be expressed as

$$
t_{c}=t_{\text {Camera }}+t_{C F}+t_{F P G A}+t_{F D}+t_{D M D},
$$

where $t_{\text {Camera }}$ is the time for frame acquisition and storage in the camera's internal recording memory; $t_{C F}$ is the time for downloading the frame from the camera to the FPGA; $t_{F P G A}$ is the time for code generating; $t_{F D}$ is the time for code transmission from the FPGA to the DMD controller; $t_{D M D}$ is the time for the DMD to set the code pattern. We estimated $t_{c}$ for setting a code with $128 \times 128$ pixels using available commercial devices as follows. For a camera with a USB 3.0 interface $(\sim 250 \mathrm{MB} / \mathrm{s})$ and a frame rate of $20 \mathrm{Kfps}$ for images of 8 -bit and $128 \times 128$ pixels, $t_{\text {Camera }}=1 / 20 \mathrm{~ms}$ and $t_{C F}=128^{2} /\left(2.5 \times 10^{8}\right) \mathrm{s}=0.07 \mathrm{~ms} . t_{F P G A}=$ $0.1 \mathrm{~ms}$ with 16513 clock cycles and a clock duration of $7.763 \mathrm{~ns}$. For a DMD with a USB 3.0 interface and a pattern rate of 30 $\mathrm{kHz}$ for 1-bit binary patterns (the DLP7000 XGA chipset, Texas Instruments $), t_{F D}=128^{2} /\left(8 \times 2.5 \times 10^{8}\right) \mathrm{s}=0.01 \mathrm{~ms}$ and $t_{D M D}=$ $1 / 30 \mathrm{~ms}$. Overall. $t_{c}=0.26 \mathrm{~ms}, t_{c}$ will be larger depending on the instruments used in the srCUP system. $t_{p}$ should not be shorter than $t_{c}$, which means the two laser pulses' maximum repetition rate is $\sim 3.8 \mathrm{KHz}$.

If $t_{p}=t_{c}=0.26 \mathrm{~ms}$, the object should not move a length longer than one pixel in $0.26 \mathrm{~ms}$. The sizes of the FoV and the corresponding image have a relationship expressed as

$$
\text { Fo } V_{x}=N_{x} d_{x} / M, \text { FoV } V_{y}=N_{y} d_{y} / M,
$$

where Fo $V_{x}$ and FoV $V_{y}$ represent the $x$ and $y$ lengths of the FoV, $N_{x}$ and $N_{y}$ represent the number of pixels of the integrated intensity image along $x$ and $y$ axes, $d_{x}$ and $d_{y}$ represent the pixel size of the image and $M$ represents the magnification of the objective lens $\mathrm{L}_{3}$. For a scene with $N_{x} \times N_{y}=128 \times 128$ pixels and $d_{x}=d_{y}=39 \mu \mathrm{m}$, Fo $V_{x}=F o V_{y}=5 / \mathrm{M} \mathrm{mm}^{2}$ and the speed of the object should be lower than $150 / \mathrm{Mmm} / \mathrm{s}$. Otherwise, the mask would block some valid signals from the object with the second pulse, leading to a loss of the spatial information.

Therefore, srCUP is suitable for the scenarios where objects move slowly $(<150 / \mathrm{Mmm} / \mathrm{s})$ to capture the transient microenvironment information in a single-shot ( $\sim 10 \mathrm{~ns}$ exposure). The signal from objects should be strong enough to guarantee a sufficient signal-to-noise ratio (SNR) of the camera images. If Poisson noise is considered and SNR $=32(30 \mathrm{~dB})$ is needed, assuming the optical system's collective efficiency is $1 \%$ and the camera's photon efficiency is $50 \%$, the signal intensity must be larger than $4 \times 10^{5}$ photons/pixel in a single-shot. However, the limitation in the intensity is not only for srCUP but also for CUP, TCUP, and other single-shot FLIM methods. The SNR can be improved by binning adjacent pixels or increasing the collective efficiency and the camera's photon efficiency.

For CUP, TCUP, and srCUP, the image reconstruction is equivalent to solving an inverse problem for compressed sensing. With the obtained 2D images, an objective function can be constructed as,

$$
\chi^{2}=\frac{1}{2}\|E-\mathbf{O} I\|^{2}+\beta \Phi(I) .
$$

Then the inverse problem is a minimization problem as [27]:

$$
\hat{I}=\arg \min _{I} \chi^{2}
$$

where $I$ is the unknown original 3D scene, $\hat{I}$ is the reconstructed scene $\left(N_{x} \times N_{y} \times N_{t}\right)$ by solving the above problem, $E$ is the measurement that contains all obtained images, $\mathbf{O}$ is the linear operator, $\Phi(I)$ is the regularization function in the form of the total variation, and $\beta$ is the regularization parameter. $E=\left[E_{\mathcal{u}}, \alpha E_{S}\right]^{T}$, where $E_{u}$ and $E_{S}$ are Image 2 and SC image, respectively, and $\alpha$ is a scaling factor. $\mathbf{O}=\left[\mathbf{O}_{u}, \alpha \mathbf{O}_{s}\right]^{T}, \mathbf{O}_{u}=\mathbf{T}, \mathbf{O}_{s}=\mathbf{T S C}$, where the linear operator $\mathbf{C}$ represents spatial encoding, $\mathbf{S}$ represents image shearing, and $\mathbf{T}$ represents spatiotemporal integration. The algorithm for solving Eq. 4 is the two-step iterative shrinkage/thresholding (TwIST) method [45].

Fig. 4 (a) summarizes the codes, normalized $E_{u}$, and $E_{s}$ used for image reconstruction with CUP, TCUP $(\alpha=4)$, and srCUP $(\alpha=4)$, respectively. For CUP and TCUP, the whole FoVs are randomly encoded as objects' spatial information is unknown. In contrast, the code for srCUP is generated with the prior spatial information upon Image 1 . For $E_{u}, C U P$ uses an empty matrix and TCUP uses an integrated intensity image, whereas srCUP uses an integrated intensity image after hard thresholding. $E_{S}$ means SC images, as shown in Row 3, Fig. 4 (a). For CUP and TCUP, the background noise is accumulated during 
the SC's shearing operation, leading to a less accurate reconstruction of the intensity decay. The precision of the recovered decay is crucial for lifetime determinations. Therefore, it is necessary to reduce the influence of noise with srCUP. Users can also disable the FPGA-controlled DMD mode (back to the traditional TCUP mode) to image fast-moving objects. However, if the background is noisy and the objects move slower $(<\sim$ $150 / M \mathrm{~mm} / \mathrm{s}$ ), srCUP would be a better choice for the benefits of relatively more precise reconstruction performances and an improved reconstruction speed.

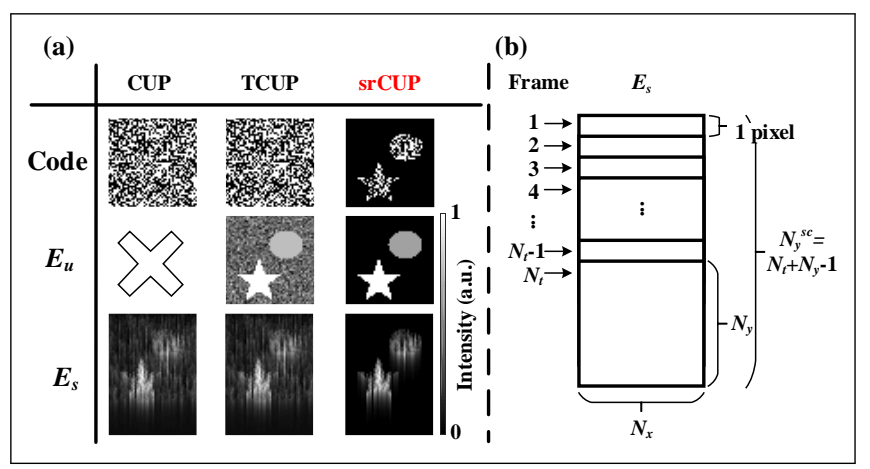

Fig. 4. (a) Illustrations of codes, normalized $E_{u}$, and $E_{s}$ used for reconstruction with CUP, TCUP, and srCUP. (b) Illustration of the relationship between the size of $E_{S}$ and the size of a reconstructed scene.

Fig. 4 (b) shows the relationship between $E_{s}$ 's size and a reconstructed scene's size. The SC image can be interpreted as an overlapping of the frames in the discrete reconstructed scene with a size of $N_{x} \times N_{y} \times N_{t}$. If the spatial interval of two adjacent frames is one pixel, then the size of $E_{s}$ is $N_{x} \times N_{y}^{s c}$ with

$$
N_{y}^{s c}=N_{t}+N_{y}-1 .
$$

$N_{t}$ is determined by the measurement window $T$, the swapping speed $v_{S C}$ and the pixel size $d$ of the SC,

$$
N_{t}=v_{s} c \times T / d,
$$

where $c$ is the speed of light. For instance, if $N_{x}=N_{y}=128, v_{s c}$ $=0.25 \mathrm{~m} / \mathrm{s}, T=10 \mathrm{~ns}$ and $d=39 \mu \mathrm{m}$, then $N_{y}^{s c}=191$ and $N_{t}=$ 64. The time resolution of the reconstructed scene is $\Delta t=T / N_{t}$ $=0.156 \mathrm{~ns}$.

Although we only discussed the TCUP system being modified with the spatial constraint for FLIM in this work, other CUP variants can also use the proposed method, such as the compressed ultrafast spectral photography (CUSP) proposed by Wang et al. [35] to improve the performances for spectral resolved FLIM.

\section{SIMULATIONS AND ANALYSIS}

A scene with nine fluorescence beads was simulated with $N_{x}$ $=N_{y}=128$. The measurement window $T$ and time resolution $\Delta t$ are $10 \mathrm{~ns}$ and $0.156 \mathrm{~ns}$, which means $N_{t}=64$ frames will be reconstructed. The fluorescence signals emitted by fluorescent beads are assumed to have a bi-exponential decay model with

$$
I(t)=A\left[q_{1} \exp \left(-t / \tau_{1}\right)+\left(1-q_{1}\right) \exp \left(-t / \tau_{2}\right)\right],
$$

where $\tau_{1}$ and $\tau_{2}\left(\tau_{1}<\tau_{2}\right)$ are two lifetime components, $A$ is the amplitude and $q_{1}$ is the proportion of the lifetime component $\tau_{1}$.
Fig. 5 shows $\log _{10}\left(E_{u}\right)$ images for three cases where

$$
E_{u}=\int I(t) d t
$$

The beads' integrated intensities in Rows $1 \sim 3$ are $I_{1}, I_{2}$, and $I_{3}$ with $I_{2}=5 I_{1}$ and $I_{3}=10 I_{1}$. The integrated intensity of the background noise randomly distributes in the range of $0 \sim I_{\text {noise }}$.

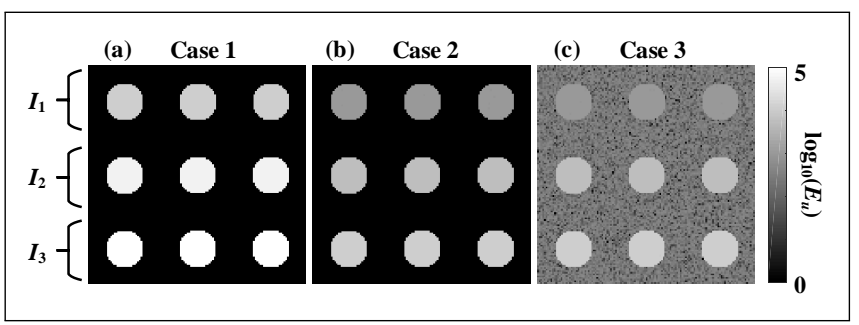

Fig. 5. $\log _{10}\left(E_{u}\right)$ images for Cases (a) 1 , (b) 2, and (c) 3 .

Table 1 summarizes $I_{1}$ and $I_{\text {noise }}$ for the three cases. Case 1 has the highest intensity without background noise; Case 2 has one order of magnitude lower intensity than Case 1 with $I_{\text {noise }}=$ 0 ; Case 3 is similar to Case 2 but with added background noise $I_{\text {noise }}=500$. Poisson noise is included in the scenes. Cases 2 and 3 serve as low intensity and high background noise scenarios compared with Case 1 to illustrate how the signal intensity and background noise affect reconstruction and lifetime determinations.

Table 1. $I_{1}$ and $I_{\text {noise }}$ for Cases $1 \sim 3$.

\begin{tabular}{ccc}
\hline Case & $I_{1}$ & $I_{\text {noise }}$ \\
\hline 1 & $10^{4}$ & 0 \\
2 & $10^{3}$ & 0 \\
3 & $10^{3}$ & 500 \\
\hline
\end{tabular}

Fig. 6 shows the $\tau_{1}$ image of the simulated scene with $1,1.5$, and $2 \mathrm{~ns}$ for the beads in Columns $1 \sim 3 . \tau_{2}$ and $q_{1}$ are assumed to be the same for the beads with $\tau_{2}=3 \mathrm{~ns}$ and $q_{1}=0.5$.

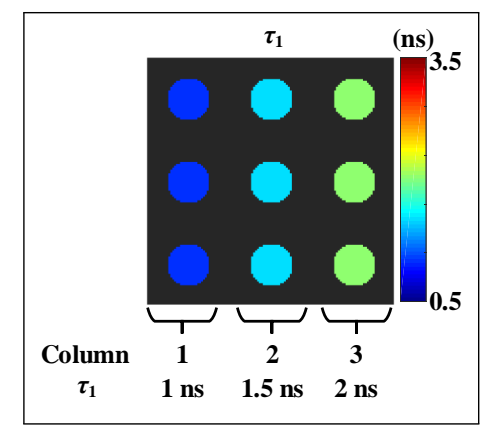

Fig. 6. $\tau_{1}$ image of the simulated scene with $1,1.5$, and $2 \mathrm{~ns}$ for the beads in Columns $1 \sim 3$.

The reconstructive performances are assessed with the mean squared error MSE of Frame $k, k=1, \ldots, N_{t}$, which is defined as

$$
\operatorname{MSE}(k)=\sum_{i=1}^{N_{x}} \sum_{j=1}^{N_{y}}[\hat{I}(i, j, k)-I(i, j, k)]^{2} / N_{x} N_{y} .
$$


Fig. 7 (a) shows the normalized $\log _{10}(M S E)$ curves of the reconstructed frames for Case 3. srCUP has the lowest MSEs for all the frames. Fig. 7 (b) shows normalized convergent $\log _{10}\left(\chi^{2}\right)$ curves with CUP, TCUP, and srCUP for Case 3. The execution times for CUP, TCUP, and srCUP are $t_{1}, t_{2}$, and $t_{3}$, respectively. $t_{3}$ is the shortest with $t_{1}=3 t_{3}$ and $t_{2}=4 t_{3} . t_{3}=5 \mathrm{~min}$ for the reconstruction executed on Matlab R2017a, 64-bit with the Intel(R) Core(TM) i5-8265U CPU (@1.60GHz).

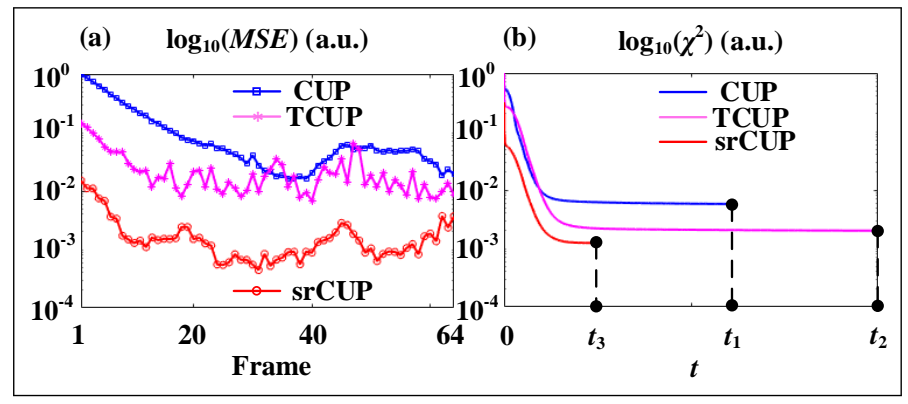

Fig. 7. (a) Normalized MSEs curves of the reconstructed frames and (b) normalized convergent $\log _{10}\left(\chi^{2}\right)$ curves with CUP, TCUP, and srCUP.

With the reconstructed decays, lifetime determination algorithms (LDA) can estimate lifetimes with different types of outputs. Fitting methods (including the least square method (LSM), maximum likelihood estimation and global fitting method [46]) can provide lifetime components. In contrast, fitting-free methods (including the integral extraction method [47], the centre-ofmass method [48], the phasor method [49], and the rapid lifetime determination method [50]) provide average lifetimes [51]. In this work, LSM is used to estimate $q_{1}, \tau_{1}$, and $\tau_{2}$ images based on a bi-exponential decay model first and then amplitude- and intensity-weighted lifetimes $\left(\tau_{A}\right.$ and $\left.\tau_{I}\right)$ are calculated with

$$
\begin{gathered}
\tau_{A}=q_{1} \tau_{1}+\left(1-q_{1}\right) \tau_{2} \\
\tau_{I}=\left[q_{1} \tau_{1}^{2}+\left(1-q_{1}\right) \tau_{2}^{2}\right] /\left[q_{1} \tau_{1}+\left(1-q_{1}\right) \tau_{2}\right] .
\end{gathered}
$$

Figs. $8(\mathrm{a} 1)-(\mathrm{a} 4)$ show the ground truth $\tau_{1}, \tau_{2}, \tau_{A}$, and $\tau_{I}$ images for Case 1 . The gray and color bars represent $\log _{10}\left(E_{u}\right)$ and $\tau_{i}, i=1,2, A, I$, respectively. Fig. 8 also shows estimated images with (b1) - (b4) CUP, (c1) - (c4) TCUP, and (d1) - (d4) srCUP for Case 1. Likewise, the gray and color bars represent $\log _{10}\left(\hat{E}_{u}\right)$ and $\hat{\tau}_{i}, i=1,2, A, I$, respectively. $\hat{\tau}_{i}$ and $\hat{E}_{u}$ are the estimated values of $\tau_{i}$ and $E_{u}$, respectively.

The accuracy and precision for lifetime determinations of the nine beads can be assessed with the relative bias $(B)$ and the coefficient of variation $(\mathrm{CV})$ defined as

$$
B(x)=|\bar{x}-x| / x, C V(x)=\sigma_{\hat{x}} / \bar{x},
$$

where $\bar{x}$ and $\sigma_{\hat{x}}$ represent the mean and the standard deviation of $\hat{x}$ with $x$ represents $\tau_{i}, i=1,2, A, I$ in a bead. Fig. 9 shows $B$ and $C V$ bar plots of the beads for Case 1 with (a1) - (a4) CUP, (b1) - (b4) TCUP and (c1) - (c4) srCUP. The horizontal axis represents the intensity and the color bars represent the beads in Columns $1 \sim 3$.

For CUP, TCUP, and srCUP, some beads have large $B$ s and $C V$ s of $\tau_{1}$ and $\tau_{2}$. In biological phenomena, it is difficult to characterize a decay with a bi-exponential model. Instead, $\tau_{A}$ and $\tau_{I}$ are estimated more robustly and are more useful in many FLIM applications, such as calculating FRET efficiency and analyzing

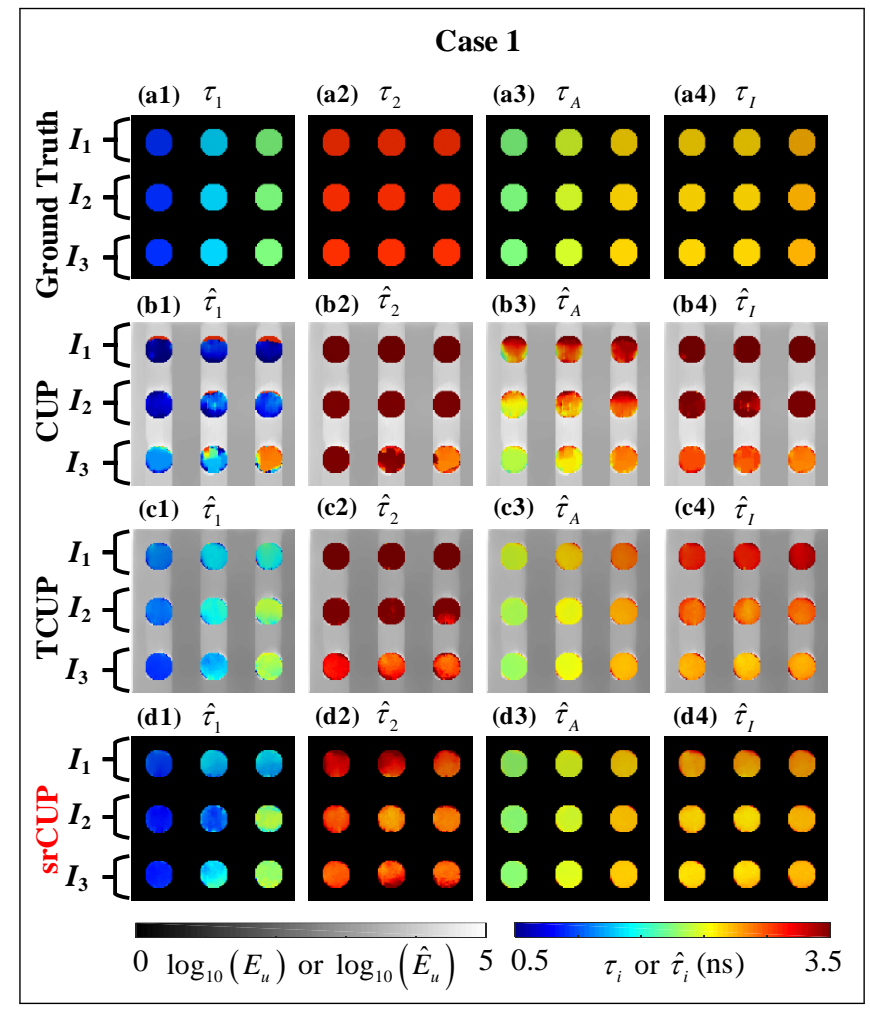

Fig. 8. (a1) - (a4) Ground truth $\tau_{1}, \tau_{2}, \tau_{A}$, and $\tau_{I}$ images for Case 1. Estimated images with (b1) - (b4) CUP, (c1) - (c4) TCUP, and (d1) - (d4) srCUP for Case 1 . The gray bar represents $\log _{10}\left(E_{u}\right)$ or $\log _{10}\left(\hat{E}_{u}\right)$. The color bar represents $\tau_{i}$ or $\hat{\tau}_{i}, i=1,2, A, I$.

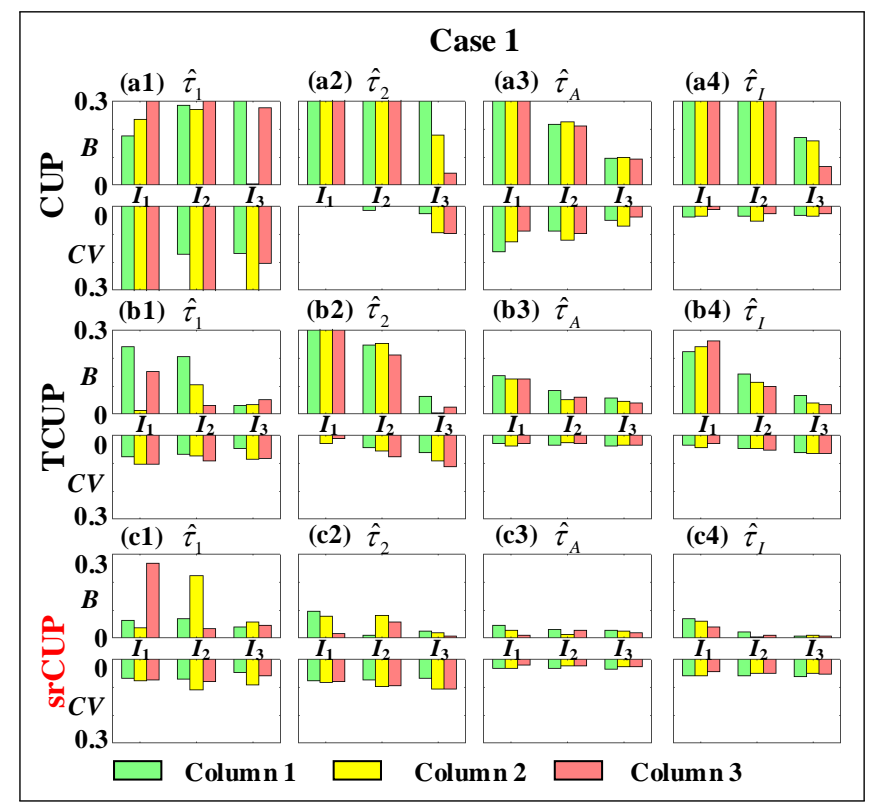

Fig. 9. $B$ and $C V$ bar plots of the beads for Case 1 with (a1) (a4) CUP, (b1) - (b4) TCUP and (c1) - (c4) srCUP. The horizontal axis represents the intensity. The color bars represent the beads in Columns $1 \sim 3$. 
quenching dynamics [51]. CUP fails to estimate $\tau_{A}$ and $\tau_{I}$ for most of the beads especially for the ones with lower intensities ( $I_{1}$ and $I_{2}$ ); TCUP is able to estimate $\hat{\tau}_{A}$ and $\hat{\tau}_{I}$ for Case 1 successfully with $B\left(\tau_{A}\right)<13 \%, C V\left(\tau_{A}\right)<3 \%, B\left(\tau_{I}\right)<25 \%$, and $C V\left(\tau_{I}\right)<7 \%$; srCUP has the best performances with $B\left(\tau_{A}\right)<$ $4 \%, C V\left(\tau_{A}\right)<3 \%, B\left(\tau_{I}\right)<7 \%$, and $C V\left(\tau_{I}\right)<6 \%$.

Fig. 10 shows (a1) $-(\mathrm{a} 4)$ the ground truth $\tau_{1}, \tau_{2}, \tau_{A}$, and $\tau_{I}$ images and estimated images with (b1) - (b4) CUP, (c1) - (c4) TCUP, and (d1) - (d4) srCUP for Case 2 . Fig. 11 shows $B$ and $C V$ bar plots of the beads for Case 2 with (a1) - (a4) CUP, (b1) (b4) TCUP and (c1) - (c4) srCUP.

Fig. 12 shows (a1) $-(\mathrm{a} 4)$ the ground truth $\tau_{1}, \tau_{2}, \tau_{A}$, and $\tau_{I}$ images and estimated images with (b1) - (b4) CUP, (c1) - (c4) TCUP, and (d1) - (d4) srCUP for Case 3 . Fig. 13 shows $B$ and $C V$ bar plots of the beads for Case 3 with (a1) - (a4) CUP, (b1) (b4) TCUP and (c1) - (c4) srCUP.

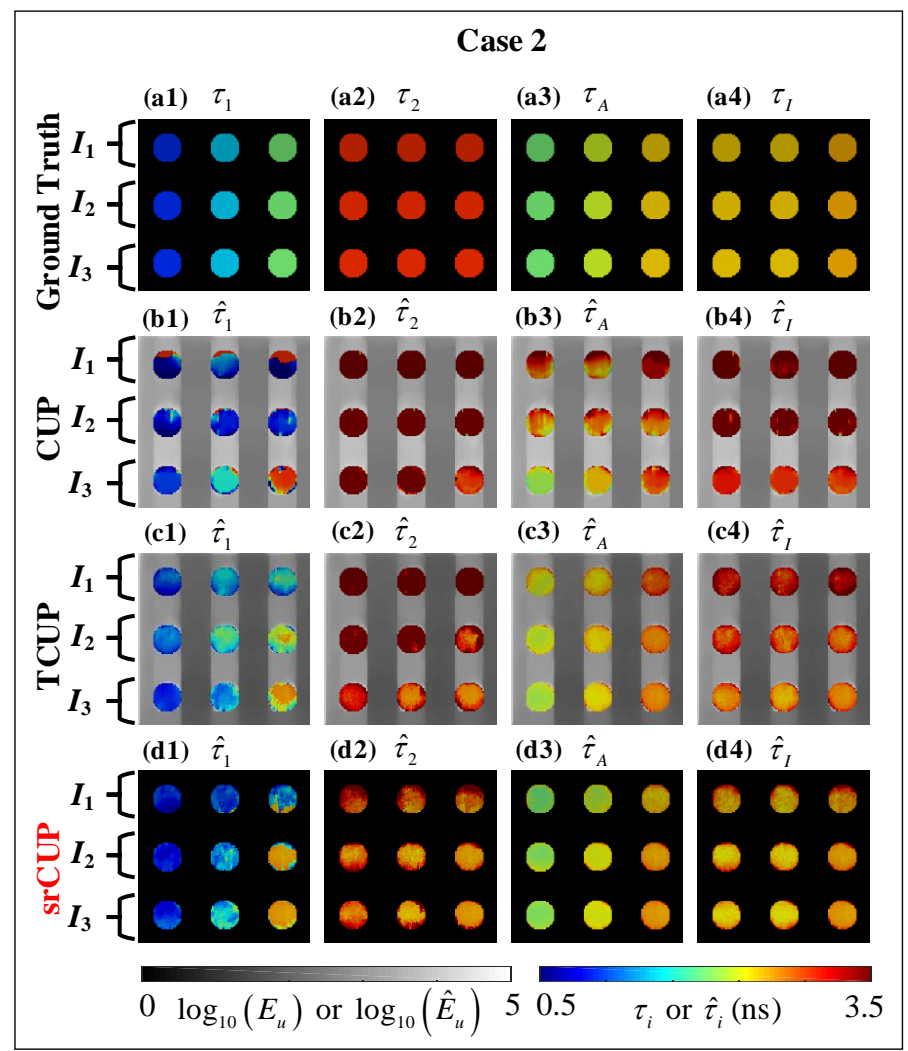

Fig. 10. (a1) - (a4) Ground truth $\tau_{1}, \tau_{2}, \tau_{A}$, and $\tau_{I}$ images for Case 2. Estimated images with (b1) - (b4) CUP, (c1) - (c4) TCUP, and (d1) - (d4) srCUP for Case 2.

For Cases 2 and 3, CUP fails to estimate the four parameters; TCUP is sensitive to the intensity and background noise and its performances are much worse than Case 1. Although srCUP has higher $B \mathrm{~s}$ and $C V \mathrm{~s}$ of $\tau_{1}$ and $\tau_{2}$ than the results for Case 1 , it estimates $\tau_{A}$ and $\tau_{I}$ robustly with a slightly increased $B \mathrm{~s}$ and $C V \mathrm{~s}\left(B\left(\tau_{A}\right)<7 \%, C V\left(\tau_{A}\right)<7 \%, B\left(\tau_{I}\right)<10 \%\right.$, and $C V\left(\tau_{I}\right)$ $<11 \%)$ and in general outperforms CUP and TCUP.

For Cases 1 and 2, even though the background noise is negligible $(\sim 0)$, srCUP still performs the best as the spatial coding operator $\mathbf{C}$ is spatially constrained in Eq. 3 (only the pixels in objects are optimized). CUP and TCUP optimize all the pixels in the FoV which leads to reconstructed noise outside the objects and results in a reconstruction loss within the objects.

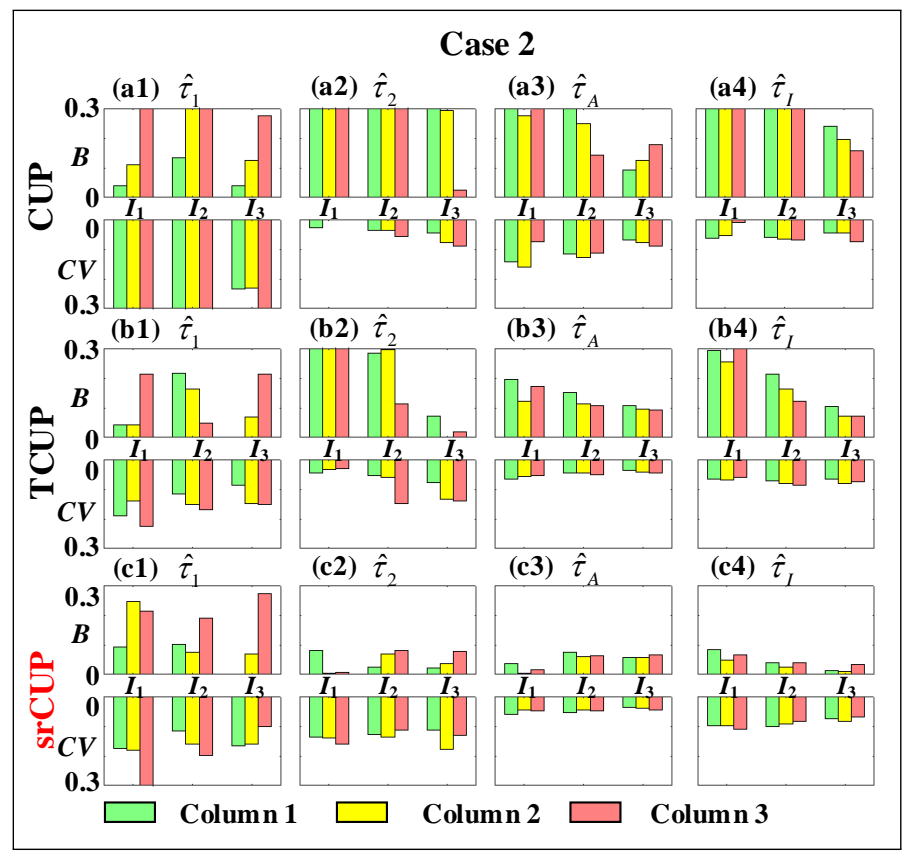

Fig. 11. $B$ and $C V$ bar plots of the beads for Case 2 with (a1) (a4) CUP, (b1) - (b4) TCUP and (c1) - (c4) srCUP.

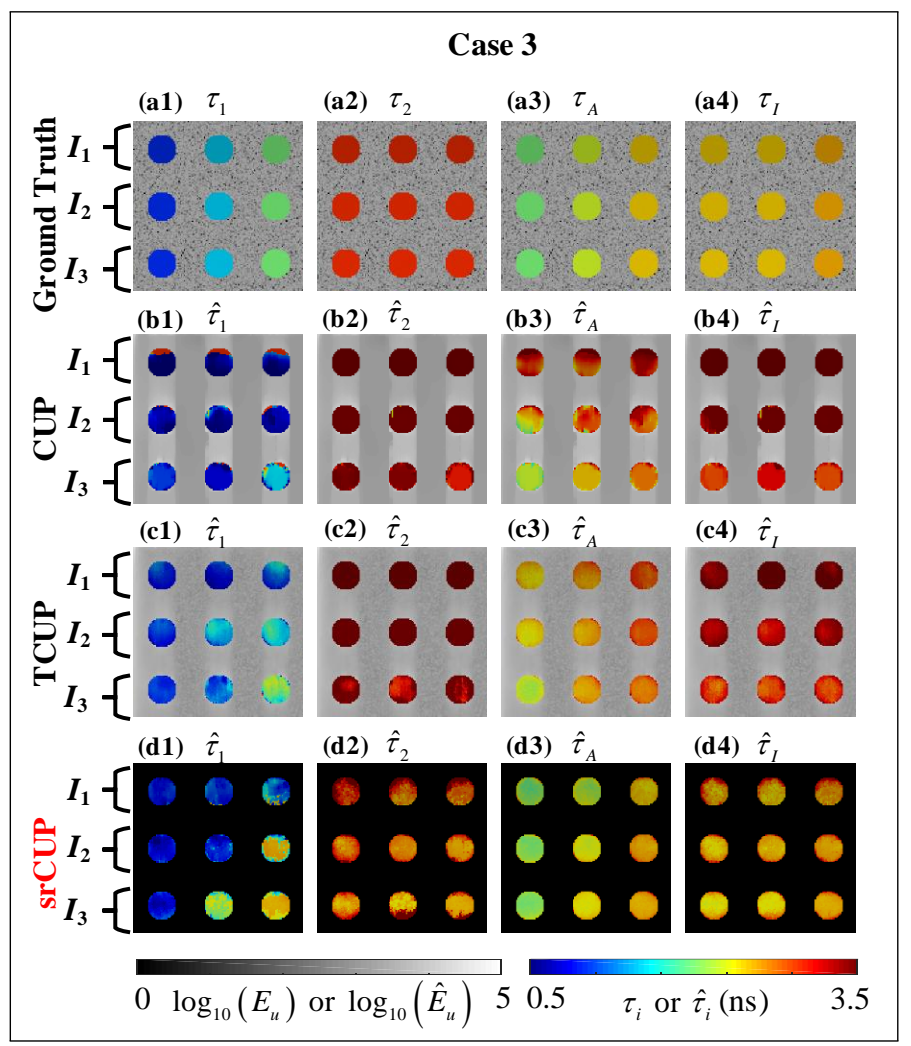

Fig. 12. (a1) - (a4) Ground truth $\tau_{1}, \tau_{2}, \tau_{A}$, and $\tau_{I}$ images for Case 3. Estimated images with (b1) - (b4) CUP, (c1) - (c4) TCUP, and (d1) - (d4) srCUP for Case 3. 


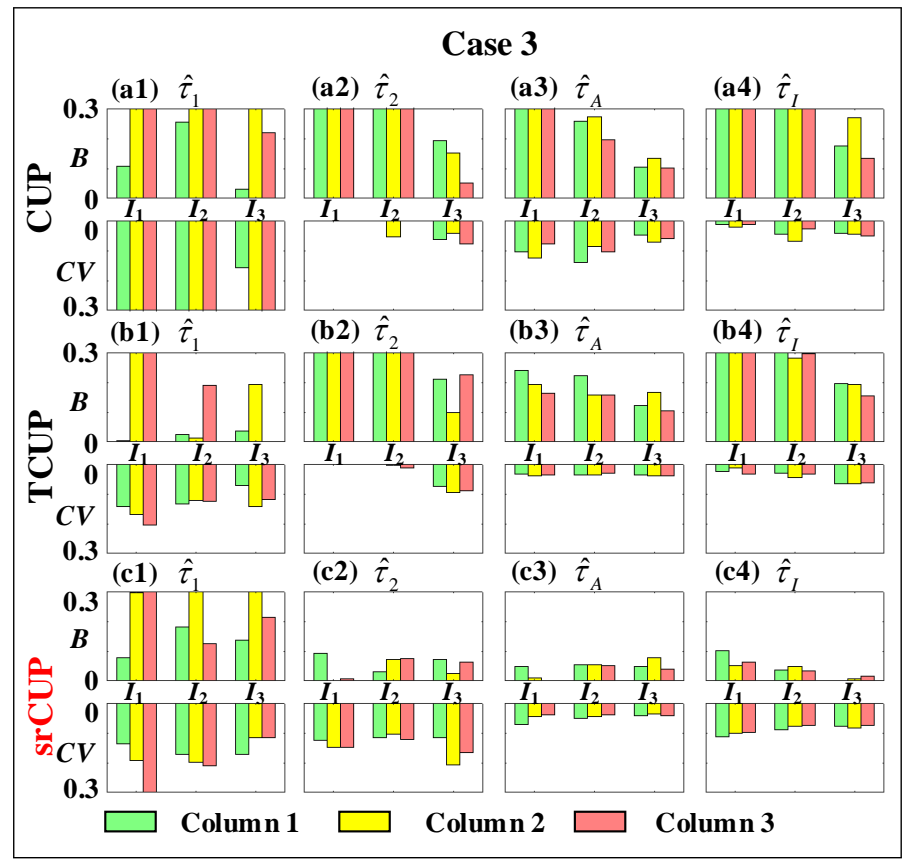

Fig. 13. $B$ and $C V$ bar plots of the beads for Case 3 with (a1) (a4) CUP, (b1) - (b4) TCUP and (c1) - (c4) srCUP.

For Case 3, the background noise is also reconstructed with CUP and TCUP, as shown in Figs. 12 (b1) - (b4) and (c1) - (c4), which is unnecessary as the background noise will be blocked during lifetime determinations and only the lifetimes of the samples are desired for FLIM applications. srCUP blocks the noise in the data acquisition process and reduces the noise's influence on the signal reconstruction, as shown in Figs. 12 (d1) - (d4).

\section{CONCLUSION}

We presented a single-shot fluorescence lifetime imaging (FLIM) method based on the compressed ultrafast photography (CUP), named space-restricted CUP (srCUP). Compared to CUP and its variant TCUP, srCUP is more suitable for FLIM where objects move slowly $(<\sim 150 / M \mathrm{~mm} / \mathrm{s}, M$ is the magnification of the objective lens) and the intensity varies rapidly in a short time window $(\sim 10 \mathrm{~ns})$. Synthetic datasets were used to simulate 3D $(x, y, t)$ scenes. The performances of srCUP are theoretically explored and compared with CUP and TCUP for three cases under different intensity and background noise conditions. With reconstructed scenes, lifetime parameters including lifetime components and amplitude- and intensity-weighted average lifetimes $\left(\tau_{A}\right.$ and $\left.\tau_{I}\right)$ are estimated with the least square method based on a bi-exponential decay model. The results show that average lifetime estimations are more reliable than lifetime component extractions. For the cases where targets having a low intensity or high background noise, CUP and TCUP fail to estimate $\tau_{A}$ and $\tau_{I}$ images. In contrast, srCUP succeeds with the best accuracy and precision for lifetime determinations with a relative bias less than $7 \%$ and a coefficient of variation less than $7 \%$ for $\tau_{A}$ and a relative bias less than $10 \%$ and a coefficient of variation less than $11 \%$ for $\tau_{I}$. In addition, the reconstruction time of srCUP is twoand three-fold faster than CUP and TCUP, respectively. Therefore, we believe srCUP is a useful single-shot FLIM method to improve the original CUP and TCUP in FLIM applications.

\section{FUNDING}

Natural Science Basic Research Plan in Shaanxi Province of China (2019JQ-930); National Natural Science Foundation of China (71705255).

\section{ACKNOWLEDGMENTS}

We thank Zhenya Zang for his help in estimating the time for code generation with FPGA. We would like to acknowledge the support from the China Scholarship Council.

\section{DISCLOSURES}

Disclosures. The authors declare no conflicts of interest.

\section{REFERENCES}

1. J. R. Lakowicz, Principles of Fluorescence Spectroscopy (Springer, 2006).

2. R. Datta, T. M. Heaster, J. T. Sharick, A. A. Gillette, and M. C. Skala, "Fluorescence lifetime imaging microscopy: fundamentals and advances in instrumentation, analysis, and applications," J. Biomed. Opt. 25, 071203 (2020).

3. N. Ma, N. R. de Mochel, P. D. Pham, T. Y. Yoo, K. W. Y. Cho, and M. A. Digman, "Label-free assessment of pre-implantation embryo quality by the Fluorescence Lifetime Imaging Microscopy (FLIM)-phasor approach," Sci. Reports 9, 13206 (2019).

4. S. J. Lee, Y. Chen, B. Lodder, and B. L. Sabatini, "Monitoring Behaviorally Induced Biochemical Changes Using Fluorescence Lifetime Photometry," Front. Neurosci. 13, 766 (2019).

5. W. Becker, "Fluorescence Lifetime Imaging - Applications and Instrumental Principles," in Encyclopedia of Cell Biology, vol. 2 (Elsevier, 2016), pp. 107-120.

6. A. Ehn, O. Johansson, J. Bood, A. Arvidsson, B. Li, and M. Aldén, "Fluorescence lifetime imaging in a flame," Proc. Combust. Inst. 33, 807-813 (2011).

7. A. Ehn, O. Johansson, A. Arvidsson, M. Aldén, and J. Bood, "Singlelaser shot fluorescence lifetime imaging on the nanosecond timescale using a Dual Image and Modeling Evaluation algorithm," Opt. Express 20, 3043-3056 (2012).

8. M. Jonsson, A. Ehn, M. Christensen, M. Aldén, and J. Bood, "Simultaneous one-dimensional fluorescence lifetime measurements of $\mathrm{OH}$ and CO in premixed flames," Appl. Phys. B 115, 35-43 (2014).

9. A. Ehn, J. Zhu, X. Li, and J. Kiefer, "Advanced Laser-Based Techniques for Gas-Phase Diagnostics in Combustion and Aerospace Engineering," Appl. Spectrosc. 71, 341-366 (2017).

10. Y. Ning, S. Cheng, J.-X. Wang, Y.-W. Liu, W. Feng, F. Li, and J.-L. Zhang, "Fluorescence lifetime imaging of upper gastrointestinal $\mathrm{pH}$ in vivo with a lanthanide based near-infrared $\tau$ probe," Chem. Sci. 10, 4227-4235 (2019).

11. H.-J. Lin, P. Herman, and J. R. Lakowicz, "Fluorescence lifetimeresolved pH imaging of living cells," Cytom. A. 52, 77-89 (2003).

12. N. Inada, N. Fukuda, T. Hayashi, and S. Uchiyama, "Temperature imaging using a cationic linear fluorescent polymeric thermometer and fluorescence lifetime imaging microscopy," Nat. Protoc. 14, 1293-1321 (2019).

13. K. Okabe, N. Inada, C. Gota, Y. Harada, T. Funatsu, and S. Uchiyama, "Intracellular temperature mapping with a fluorescent polymeric thermometer and fluorescence lifetime imaging microscopy," Nat. Commun. 3, 705 (2012).

14. M. K. Kuimova, G. Yahioglu, J. A. Levitt, and K. Suhling, "Molecular rotor measures viscosity of live cells via fluorescence lifetime imaging," J. Am. Chem. Soc. 130, 6672-6673 (2008).

15. X. Peng, Z. Yang, J. Wang, J. Fan, Y. He, F. Song, B. Wang, S. Sun, J. Qu, J. Qi, and M. Yan, "Fluorescence Ratiometry and Fluorescence Lifetime Imaging: Using a Single Molecular Sensor for Dual Mode Imaging of Cellular Viscosity," J. Am. Chem. Soc. 133, 6626-6635 (2011). 
16. J. Neef, N. T. Urban, T.-L. Ohn, T. Frank, P. Jean, S. W. Hell, K. I. Willig, and T. Moser, "Quantitative optical nanophysiology of $\mathrm{Ca} 2+$ signaling at inner hair cell active zones," Nat. Commun. 9, 290 (2018).

17. K. Zheng, T. P. Jensen, and D. A. Rusakov, "Monitoring intracellular nanomolar calcium using fluorescence lifetime imaging," Nat. Protoc. 13, 581-597 (2018).

18. N. Hirmiz, A. Tsikouras, E. J. Osterlund, M. Richards, D. W. Andrews, and Q. Fang, "Highly Multiplexed Confocal Fluorescence Lifetime Microscope Designed for Screening Applications," IEEE J. Sel. Top. Quantum Electron. 27, 1-9 (2020).

19. J. A. Levitt, S. P. Poland, N. Krstajic, K. Pfisterer, A. Erdogan, P. R. Barber, M. Parsons, R. K. Henderson, and S. M. Ameer-Beg, "Quantitative real-time imaging of intracellular FRET biosensor dynamics using rapid multi-beam confocal FLIM," Sci. Reports 10, 5146 (2020).

20. C. Poudel, I. Mela, and C. F. Kaminski, "High-throughput, multiparametric, and correlative fluorescence lifetime imaging," Methods Appl. Fluoresc. 8, 024005 (2020).

21. L. M. Hirvonen and K. Suhling, "Wide-field TCSPC: methods and applications," Meas. Sci. Technol. 28, 012003 (2017).

22. W. Becker, "Fluorescence lifetime imaging by multi-dimensional time correlated single photon counting," Med. Photonics 27, 41-61 (2015).

23. K. Suhling, L. M. Hirvonen, W. Becker, S. Smietana, H. Netz, J. Milnes, T. Conneely, A. L. Marois, O. Jagutzki, F. Festy, Z. Petrášek, and A. Beeby, "Wide-field time-correlated single photon counting-based fluorescence lifetime imaging microscopy," Nucl. Instruments Methods Phys. Res. Sect. A: Accel. Spectrometers, Detect. Assoc. Equip. 942, 162365 (2019).

24. Y. Li, H. Jia, S. Chen, J. Tian, L. Liang, F. Yuan, H. Yu, and D. D.-U. Li, "Single-shot time-gated fluorescence lifetime imaging using three-frame images," Opt. Express 26, 17936-17947 (2018).

25. L. Liu, Y. Li, L. Sun, H. Li, X. Peng, and J. Qu, "Fluorescence lifetime imaging microscopy using a streak camera," in Progress in Biomedical Optics and Imaging - Proceedings of SPIE, vol. 8948 A. Periasamy, P. T. C. So, and K. König, eds. (2014), p. 89482L.

26. L. Camborde, A. Jauneau, C. Brière, L. Deslandes, B. Dumas, and E. Gaulin, "Detection of nucleic acid-protein interactions in plant leaves using fluorescence lifetime imaging microscopy," Nat. Protoc. 12, 19331950 (2017)

27. L. Gao, J. Liang, C. Li, and L. V. Wang, "Single-shot compressed ultrafast photography at one hundred billion frames per second," Nature 516, 74-77 (2014).

28. J. Liang, L. Zhu, and L. V. Wang, "Single-shot real-time femtosecond imaging of temporal focusing," Light. Sci. \& Appl. 7, 42 (2018).

29. J. Liang, P. Wang, L. Zhu, and L. V. Wang, "Single-shot stereopolarimetric compressed ultrafast photography for light-speed observation of high-dimensional optical transients with picosecond resolution," Nat. Commun. 11, 5252 (2020).

30. Y. Lai, Y. Xue, C. Côté, X. Liu, A. Laramée, N. Jaouen, F. Légaré, L. Tian, and J. Liang, "Single-Shot Ultraviolet Compressed Ultrafast Photography," Laser \& Photonics Rev. 14, 2000122 (2020).

31. D. Qi, C. Yang, F. Cao, J. Liang, Y. He, Y. Yang, T. Jia, Z. Sun, and S. Zhang, "Compressed Ultrafast Electron Diffraction Imaging Through Electronic Encoding," Phys. Rev. Appl. 10, 054061 (2018).

32. T. Kim, J. Liang, L. Zhu, and L. V. Wang, "Picosecond-resolution phasesensitive imaging of transparent objects in a single shot," Sci. Adv. 6 , eaay6200 (2020).

33. J. V. Thompson, J. D. Mason, H. T. Beier, and J. N. Bixler, "High speed fluorescence imaging with compressed ultrafast photography," SPIE Proc. 10076, High-Speed Biomed. Imaging Spectrosc. Toward Big Data Instrumentation Manag. II 10076, 1007613 (2017).

34. Y. Ma, Y. Lee, C. Best-Popescu, and L. Gao, "High-speed compressedsensing fluorescence lifetime imaging microscopy of live cells," bioRxiv (2020).

35. P. Wang, J. Liang, and L. V. Wang, "Single-shot ultrafast imaging attaining 70 trillion frames per second," Nat. Commun. 11, 2091 (2020).

36. D. Qi, S. Zhang, C. Yang, Y. He, F. Cao, J. Yao, P. Ding, L. Gao, T. Jia, J. Liang, Z. Sun, and L. V. Wang, "Single-shot compressed ultrafast photography: a review," Adv. Photonics 2, 014003 (2020).
37. D. D.-U. Li, J. Arlt, D. Tyndall, R. Walker, J. Richardson, D. Stoppa, E. Charbon, and R. K. Henderson, "Video-rate fluorescence lifetime imaging camera with CMOS single-photon avalanche diode arrays and high-speed imaging algorithm," J. Biomed. Opt. 16, 096012 (2011).

38. W. Becker, L. M. Hirvonen, J. Milnes, T. Conneely, O. Jagutzki, H. Netz, S. Smietana, and K. Suhling, "A wide-field TCSPC FLIM system based on an MCP PMT with a delay-line anode," Rev. Sci. Instruments 87 , 093710 (2016).

39. L. Parmesan, N. A. Dutton, N. J. Calder, L. A. Grant, and R. K. Henderson, "A 256x256 SPAD array with in-pixel Time to Amplitude Conversion for Fluorescence Lifetime Imaging Microscopy," Int. Image Sens. Work. pp. 8-11 (2015).

40. R. M. Field, S. Realov, and K. L. Shepard, "A $100 \mathrm{fps,} \mathrm{Time-Correlated}$ Single-Photon-Counting-Based Fluorescence-Lifetime Imager in 130 nm CMOS," IEEE J. Solid-State Circuits 49, 867-880 (2014).

41. Wolfgang Becker, Advanced Time-Correlated Single Photon Counting Techniques (Springer, Heidelberg, 2005)

42. D. S. Elson, I. Munro, J. Requejo-Isidro, J. McGinty, C. Dunsby, N. Galletly, G. W. Stamp, M. A. A. Neil, M. J. Lever, P. A. Kellett, A. DymokeBradshaw, J. Hares, and P. M. W. French, "Real-time time-domain fluorescence lifetime imaging including single-shot acquisition with a segmented optical image intensifier," New J. Phys. 6, 180 (2004).

43. S. P. Chan, Z. J. Fuller, J. N. Demas, and B. A. DeGraff, "Optimized Gating Scheme for Rapid Lifetime Determinations of Single-Exponential Luminescence Lifetimes," Anal. Chem. 73, 4486-4490 (2001).

44. R. M. Ballew and J. N. Demas, "An error analysis of the rapid lifetime determination method for the evaluation of single exponential decays," Anal. Chem. 61, 30-33 (1989).

45. J. M. Bioucas-Dias and M. A. Figueiredo, "A new TwIST: Two-step iterative shrinkage/thresholding algorithms for image restoration," IEEE Transactions on Image Process. 16, 2992-3004 (2007).

46. S. Pelet, M. Previte, L. Laiho, and P. C. So, "A Fast Global Fitting Algorithm for Fluorescence Lifetime Imaging Microscopy Based on Image Segmentation," Biophys. J. 87, 2807-2817 (2004).

47. D.-U. Li, R. Walker, J. Richardson, B. Rae, A. Buts, D. Renshaw, and R. Henderson, "Hardware implementation and calibration of background noise for an integration-based fluorescence lifetime sensing algorithm," J. Opt. Soc. Am. A 26, $804-814$ (2009).

48. D.-U. Li, B. Rae, R. Andrews, J. Arlt, and R. Henderson, "Hardware implementation algorithm and error analysis of high-speed fluorescence lifetime sensing systems using center-of-mass method," J. Biomed. Opt. 15, 017006 (2010).

49. G. Yahav, E. Barnoy, N. Roth, L. Turgeman, and D. Fixler, "Referenceindependent wide field fluorescence lifetime measurements using Frequency-Domain (FD) technique based on phase and amplitude crossing point," J. Biophotonics 10, 1198-1207 (2017).

50. C. Moore, S. P. Chan, J. N. Demas, and B. A. DeGraff, "Comparison of Methods for Rapid Evaluation of Lifetimes of Exponential Decays," Appl. Spectrosc. 58, 603-607 (2004).

51. Y. Li, S. Natakorn, Y. Chen, M. Safar, M. Cunningham, J. Tian, and D. D.u. Li, "Investigations on Average Fluorescence Lifetimes for Visualizing Multi-Exponential Decays," Front. Phys. 8, 576862 (2020). 